\title{
Rethinking EHR interfaces to reduce click fatigue and physician burnout
}

Cite as: CMAJ 2018 August 20;190:E994-5. doi: 10.1503/cmaj.109-5644

Posted on cmajnews.com on July 31, 2018.

A Ithough electronic health records (EHR) offer many benefits, they have also become an administrative burden for busy doctors and have been linked to physician burnout. Some health care institutions are looking at practical ways to address this problem and reduce "click fatigue" in medical practice so doctors can spend more time with patients and less time with screens.

The Yale School of Medicine, for example, has turned its attention to EHR inefficiencies and is exploring better ways to enter and retrieve patient data. According to Dr. Allen Hsiao, chief medical information officer at Yale, "One of the things we realized is that there is still a limitation to the keyboard-and-mouse user interface. Anything we can do to tackle that can make a big difference and help take clicks away for physicians to complete their work."

Allowing doctors to log into EHRs using badges, instead of typing usernames and passwords up to 140 times daily, was found to save as much as 20 minutes a day. Using voice-recognition software to interact with EHRs instead of the keyboard-and-mouse interface led to a $50 \%$ reduction in the time it took to complete and close patient encounters. Using virtual scribes (people who listen in and document patient encounters from remote locations) was the third major initiative Yale tested. The combination of improved voice-recognition software and virtual scribes was found to reduce the time doctors spent on documentation.

According to a 2017 study, $70 \%$ of doctors using EHRs attribute the bulk of their administrative burden to the software. Doctors' opinion of EHRs improved, however, when their medical institutions made efforts to optimize how the software is used. More than half of physicians using optimized EHRs reported that it saved them time, compared to only $13 \%$ of those who worked in environments that didn't optimize the software.

Another recent study found that the quality of documentation by medical residents decreased as the number of dialog boxes they had to open to record information increased, which the authors attributed to mouse-click fatigue. "This phenomenon affected all residents regardless of year in training." The authors also suggested that click fatigue was similar to alert fatigue, which occurs

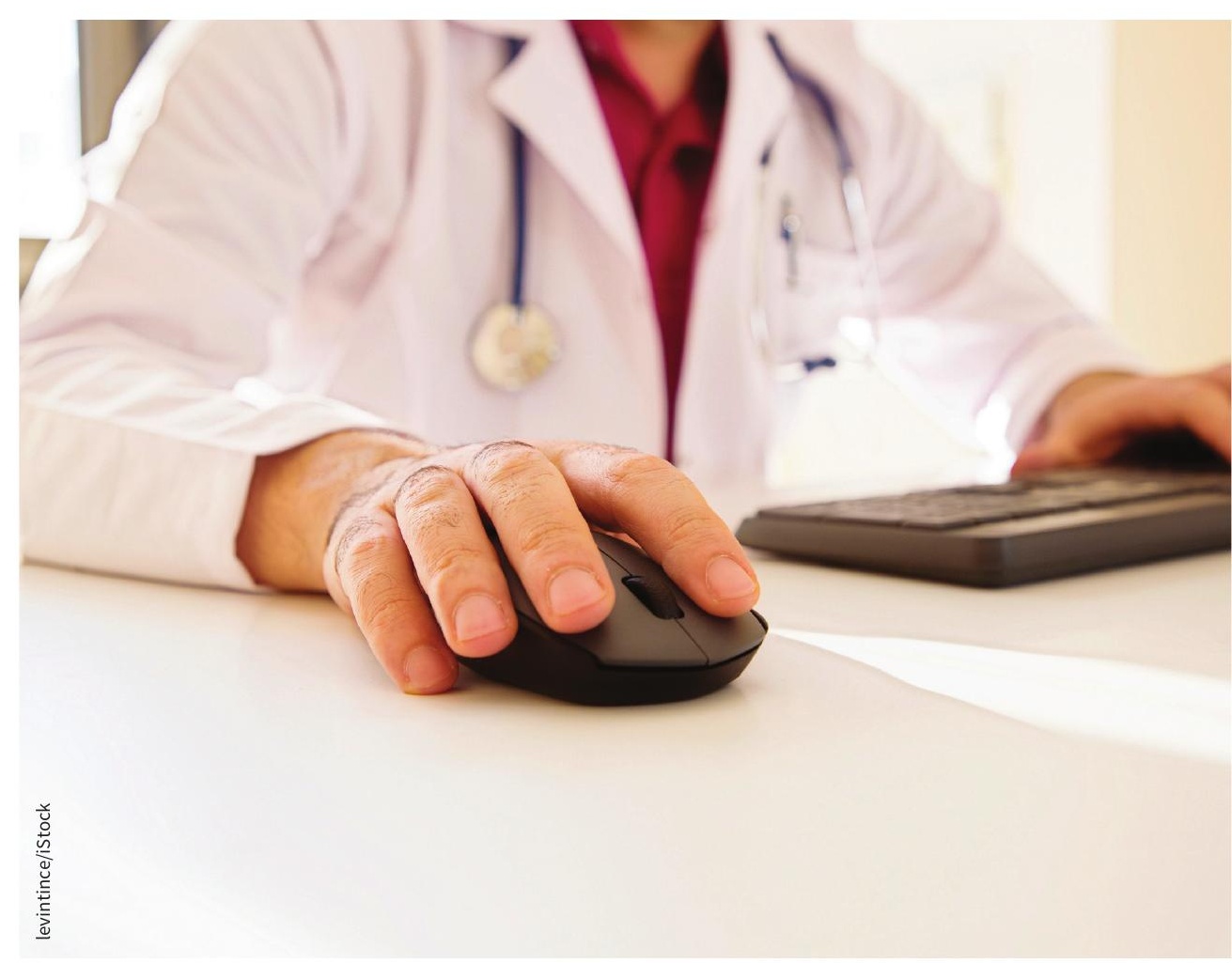

The keyboard-and-mouse interface remains a limitation to efficient EHR use. when clinicians are bombarded with insignificant reminders, a phenomenon that has been linked to an increase in patient safety hazards. "Therefore, EHR software design must be carefully evaluated for these phenomena as they can decrease physician compliance with standards of care," the authors concluded.

Some EHR vendors have taken notice of this problem and are working on better interfaces. "Click fatigue is a serious barrier to effective use of an EHR," noted Dr. Lester Wold, chief medical officer for VitalHealth Software. Poor usability, and the inefficient nature of data input in particular, is one of the most common 
complaints among EHR users, according to Wold.

One of the more ambitious projects to address this problem is being tested by Google in partnership with Stanford Medicine. Combining voice-recognition with artificial intelligence, the project aims to create a "digital scribe" - software that would listen to patient visits, parse relevant information, and automatically record that data in an EHR. The lifethreatening nature of inaccuracies, however, means this solution is unlikely to be implemented any time soon.

"This is even more of a complicated, hard problem than we originally thought," according to Dr. Steven Lin, the physician lead of the project at Stanford. "But if solved, it can potentially unshackle physicians from EHRs and bring providers back to the joys of medicine: actually interacting with patients."

Roger Collier, CMAJ 